\title{
A Influência da Integração da Cadeia de Suprimentos no Desempenho de Entrega do
}

\section{Fornecedor}

Versão do autor aceita publicada online: 11 ago. 2021

Publicado online: 30 ago. 2021

Como citar esse artigo - American Psychological Association (APA): Ribeiro, J. S. de A. N., França, R. de S., Corrêa, F., \& Ferreira, E. de P. (2021). A influência da integração da cadeia de suprimentos no desempenho de entrega do fornecedor. Exacta. https://doi.org/10.5585/exactaep.2021.18905

\section{Jurema Suely de Araújo Nery Ribeiro}

jurema.nery@gmail.com

https://orcid.org/0000-0002-6465-6020?lang=pt

Universidade Fumec

Doutora em Sistemas de Informação e Gestão do Conhecimento

Universidade Fumec

Programa de Doutorado em Sistemas de Informação e Gestão do Conhecimento

\section{Renata de Souza França}

profrenatafranca@gmail.com

Universidade UEMG

Doutora em Sistemas de Informação e Gestão do Conhecimento

\section{Fábio Corrêa}

fabiocontact@gmail.com

Doutor em Sistemas de Informação e Gestão do Conhecimento

Universidade Fumec

Programa de Doutorado em Sistemas de Informação e Gestão do Conhecimento

\section{Eric de Paula Ferreira}

eric.p.f@gmail.com

Universidade UEMG

Doutor em Sistemas de Informação e Gestão do Conhecimento

\section{Resumo}

Este trabalho busca analisar as influências da integração da cadeia de suprimentos para alcance de um desempenho superior de entrega do fornecedor. Nos procedimentos metodológicos, é delineada uma pesquisa quantitativa e descritiva. Quanto aos procedimentos, foi realizado um levantamento (survey) transversal auto-administrado on-line e impresso. 
Como ferramenta de tratamento e análise dos dados dos questionários, foi utilizado o software SPSS (Statistic Package for Social Study) e a Modelagem de Equações Estruturais com estimação por Mínimos Quadrados Parciais (PLS-SEM). Como resultado, obteve-se que o modelo proposto é válido e confiável. Os testes realizados não confirmaram a hipótese da pesquisa, pois na percepção dos respondentes, a influência da integração da cadeia de suprimentos não têm efeito positivo no desempenho de entrega do fornecedor. Já para os construtos de primeira ordem todas as dependências foram relacionadas positivamente e estatisticamente significantes. Foram apresentadas contribuições e limitações do estudo, e sugestões de trabalhos futuros.

Palavras-chave: Desempenho de entrega do fornecedor. Integração da cadeia de suprimentos. Modelo de análise.

\title{
The Influence of Supply Chain Integration on Supplier Delivery Performance
}

\begin{abstract}
This work seeks to analyze the influences of the supply chain integration to achieve a superior performance of supplier delivery. In the methodological procedures, a quantitative and descriptive research is outlined. As for the procedures, a self-administered cross-sectional survey was carried out online and in print. As a tool for the treatment and analysis of the questionnaire data, the SPSS software (Statistic Package for Social Study) and the Structural Equation Modeling with estimation by partial least squares (PLS-SEM) were used. As a result, it was found that the proposed model is valid and reliable. The tests carried out did not confirm the research hypothesis, because in the respondents' perception, the influence of the supply chain integration has no positive effect on the supplier's delivery performance. For the first order constructs, all dependencies were positively and statistically significant.
\end{abstract}

Contributions and limitations of the study and suggestions for future work were presented.

Keywords: Supplier delivery performance. Supply chain integration. Analysis model. 


\section{Introdução}

Para se manterem competitivas, as empresas devem juntar esforços para conseguirem uma maior coordenação e (Ribeiro, Ziviani, Tadeu, \& Ramos Neves, 2019), em uma abordagem chamada de "integração da cadeia de suprimentos" (Lee \& Whang, 2001). Esforços sinérgicos da integração da cadeia de suprimentos entre empresas para melhorar o desempenho são empreendidos e demonstrados em alguns estudos, sabendo-se que o desempenho do relacionamento pode ser definido como os benefícios resultantes de atividades colaborativas entre compradores e fornecedores (Villena, Choi, \& Revilla, 2012).

Porém, estar integrado com sua cadeia, por si só, não garante eficiência operacional e/ôu estratégica às empresas. É importante organizar e gerenciar as políticas e processos internos da cadeia, de forma a abranger o planejamento e a gerência de todas as atividades encarregadas no fornecimento e aquisição, conversão, e todo o gerenciamento das atividades logísticas. Por um lado, há diversos estudos mostrarem a existência de uma relação positiva entre integração da cadeia de suprimentos e desempenho organizacional individual, como os realizados por Sezen (2008), Gripa e De Carvalho (2019) entre outros. Por outro lado, estudos como dos autores Badell, Fernández, Guillén e Puigjaner (2007), Das, Narasimhan e Talluri (2006) e Swink, Narasimhan e Wang (2007) mostraram que os benefícios da integração da cadeia de suprimentos não puderam ser confirmados, mesmo com diversos benefícios atribuídos às ações de integração com outros elos da cadeia, por estudos acadêmicos e relatórios de mercado.

Em uma cadeia de suprimentos, um fator decisivo que impacta diretamente os clientes é a entrega. Trata-se de um determinante primário da satisfação do cliente. Portanto, medir e melhorar a entrega é sempre desejável para aumentar a competitividade (Viana, Neto, Añez,

\& Fernandes, 2016). A entrega, por sua própria natureza, ocorre em um ambiente dinâmico e em constante mudança, dificultando o estudo e a melhoria subsequente de um sistema de distribuição. Interrupções e atrasos nas atividades de suprimentos da cadeia logística pela ocorrência de falhas nos processos, na coordenação de atividades e na comunicação, entre outros aspectos, podem interferir negativamente no atendimento à demanda do cliente pelo fornecedor.

Entretanto, apesar de ser possível um entendimento intuitivo acerca da relação da integração entre empresas da cadeia de suprimentos com os resultados de desempenho de entrega, 
observam-se algumas lacunas e oportunidades de pesquisa. Dessa forma, faz-se importante compreender como o desenvolvimento de um ambiente propício, entre os intervenientes da cadeia de suprimentos, pode auxiliar no alcance de um desempenho de entrega ao cliente satisfatório. Nessa perspectiva, a problemática deste estudo tem a seguinte questão de pesquisa: Quais são as influências da relação entre integração da cadeia de suprimentos e desempenho de entrega do fornecedor na perspectiva do cliente?

Diante dessa problemática o objetivo geral desta pesquisa foi analisar as influências da relação entre integração da cadeia de suprimentos e desempenho superior de entrega do fornecedor. Foram desenvolvidos os seguintes objetivos específicos: i) Identificar, na literatura: os tipos de integração da cadeia de suprimentos e os indicadores mais utilizados para avaliar o desempenho de entrega dos fornecedores, sob a perspectiva do cliente; ii) verificar as relações entre: integração da cadeia de suprimentos e desempenho de entrega do fornecedor; iii) propor e validar um modelo de análise da influência dessas relações.

Para tal, este artigo foi construído em cinco seções. Na seção 2 são apresentados os conceitos basilares relacionados à Cadeia de Suprimentos Integrada e Desempenho de Entrega do Fornecedor. A metodologia, modelo proposto e hipótese da pesquisa encontram-se na seção 3 . Na seção 4 os resultados são apresentados. Por fim, as conclusões foram dispostas na seção 5.

\section{Referencial teórico}

Esta seção encontra-se dividida em duas subseções: cadeia de suprimentos integrada (2.1), desempenho de entrega dos fornecedores e seus principais indicadores (2.2).

\subsection{Cadeia de suprimentos integrada}

Para Slack, Alistair e Johnston (2018) na busca pela satisfação de seus clientes, as empresas se unem umas às outras, de forma que fornecedores, clientes e provedores externos de meios logísticos, compartilhem informações e planos necessários para tornar o canal eficiente e competitivo (cadeia de suprimentos). Lee e Whang (2001) e Abbade (2016) afirmam que a integração não pode ser completa sem um estreito vínculo de relacionamentos entre empresas, ocorrendo de acordo com: canais de comunicação (precisam ser bem definidos, com responsabilidades e funções claras); medidas de desempenho para os membros da cadeia (bem especificadas e controladas para garantir uma maior colaboração e coordenação); e incentivos 
(definindo os mecanismos pelos quais os riscos e benefícios associados aos esforços de integração são compartilhados).

Torna-se fundamental que cada empresa pertencente a uma rede de suprimentos compreenda seu papel e seu poder de contribuição para o melhor desempenho, e que possa, juntamente com seus parceiros, atuar com competitividade junto ao mercado (Bowersox, Closs, Cooper, \& Bowersox, 2016). A interação entre firmas possibilita a cooperação e o desenvolvimento regional, promove o aprendizado e a inovação, melhorando o desempenho das empresas participantes, aumentando a produtividade (Castells, 2002; Choo, 2003).

Na visão de Scandiuzzi (2011), o tema Integração da Cadeia de Suprimentos ainda merece atenção e estudos mais aprofundados. Em seus estudos, este autor verificou que alguns autores destacam a necessidade de conhecer os reais benefícios trazidos pela integração da cadeia de suprimentos, pois, apesar de muitos estudos demonstrarem possíveis benefícios gerados com tal prática, vários outros estudos não puderam comprovar tais resultados, trazendo como questionamento se os benefícios da integração da cadeia seriam "mais teoria do que realidade".

Os relacionamentos colaborativos entre os membros de uma cadeia de suprimentos estão suscetíveis a riscos, e podem falhar em atender aos anseios e necessidades dos intervenientes da cadeia de suprimentos (Da Silva, 2019; Ribeiro, 2019). Algumas causas possíveis podem estar relacionadas a aspectos metodológicos na mensuração dos construtos integração da cadeia e desempenho de entrega, uma vez que a integração da cadeia de suprimentos requer, como princípio de gestão incorporada às estratégias da organização, o monitoramento do desempenho do fornecedor (Bowersox et al., 2016). Este monitoramento deverá ocorrer pela adoção de indicadores de desempenho da cadeia de suprimentos, sendo úteis ao controlar tanto a qualidade das atividades logísticas internas à empresa como as atividades logísticas externas, ou seja, dos fornecedores.

\subsection{Desempenho de entrega dos fornecedores e seus principais indicadores}

O desempenho da cadeia de suprimentos significa o grau de eficiência e eficácia com que as empresas da cadeia atendem às necessidades logísticas de seus clientes (produto certo no lugar certo, no momento certo e nas quantidades desejadas), quando comparadas com as suas metas. O trabalho integrado e coordenado de todos os elos da cadeia de suprimentos requer, 
como princípio de gestão incorporada às estratégias da organização, um monitoramento do desempenho do fornecedor. Somente através da mensuração do desempenho é possível avaliar se as operações estão alcançando as metas pretendidas (Bowersox et al., 2016) e, assim, destinar e monitorar os recursos logísticos (Ribeiro, 2019).

Alguns esforços sinérgicos entre empresas para melhorar o desempenho de entrega são empreendidos, sabendo-se que o resultado do relacionamento pode ser definido como os benefícios advindos de atividades colaborativas entre compradores e fornecedores (CSCMP, 2019; Villena et al., 2012), devido ao impacto direto que a pontualidade da entrega tem na satisfação do cliente (Garcia-Arca; Prado-Prado; Fernándes-Gonzáles, 2018), de forma a gerar vantagem competitiva ao oferecer um produto ou serviço que, além de atender às necessidades e expectativas do cliente, proporcione a ele valor intrínseco (Cosentino, Vidal \& Virgillito, 2011; Ribeiro, Calijorne, Jurza, \& Ziviani, 2018).

Para chegar ao resultado do nível de serviço de entrega, é preciso acompanhar alguns indicadores que avaliam o serviço prestado pelos fornecedores. Alguns dos principais indicadores que atuam no monitoramento das atividades logística externas, ou seja, dos fornecedores foram elucidados no Quadro 1.

Quadro 1 - Indicadores de Desempenho Logístico Externo

\begin{tabular}{|l|l|l|}
\hline \multicolumn{1}{|c|}{$\begin{array}{c}\text { Indicador de } \\
\text { desempenho }\end{array}$} & \multicolumn{1}{|c|}{ Descrição } & \multicolumn{1}{c|}{ Cálculo } \\
\hline $\begin{array}{l}\text { Atendimento do pedido } \\
\text { realizado }\end{array}$ & $\begin{array}{l}\text { Reflete se o fornecedor está entregando a } \\
\text { quantidade de produtos solicitados }\end{array}$ & $\begin{array}{l}\mathrm{N}^{\circ} \text { produtos entregues / } \mathrm{n}^{\circ} \text { produtos } \\
\text { pedidos *100 }\end{array}$ \\
\hline $\begin{array}{l}\text { Entregas realizadas dentro } \\
\text { do prazo negociado OTD } \\
\text { (On Time Delivery) }\end{array}$ & $\begin{array}{l}\text { Calcula a taxa de entregas realizadas } \\
\text { dentro do prazo negociado com o } \\
\text { fornecedor. }\end{array}$ & $\begin{array}{l}\text { OTD = (Número de entregas no } \\
\text { prazo / Número total de entregas) x } \\
100 \%\end{array}$ \\
\hline $\begin{array}{l}\text { Entregas devolvidas } \\
\text { parcial ou integralmente }\end{array}$ & $\begin{array}{l}\text { Corresponde às entregas devolvidas } \\
\text { parcial ou integralmente devido à alguma } \\
\text { falha não aceitável do fornecedor. }\end{array}$ & $\begin{array}{l}\text { Entregas devolvidas parcial ou } \\
\text { integralmente / Total de entregas } \\
\text { recebidas (aceitas + devolvidas) }\end{array}$ \\
\hline $\begin{array}{l}\text { Recebimento de produtos } \\
\text { dentro das especificações } \\
\text { de qualidade }\end{array}$ & $\begin{array}{l}\text { Corresponde a quantidade de produtos } \\
\text { que foram entregues dentro das } \\
\text { especificações de qualidade acordadas } \\
\text { previamente com o fornecedor. }\end{array}$ & $\begin{array}{l}\text { Produtos recebidos dentro das } \\
\text { especificações de qualidade } \\
\text { acordadas com o fornecedor / Total } \\
\text { de produtos aceitos *100 }\end{array}$ \\
\hline $\begin{array}{l}\text { Tempo de entrega dos } \\
\text { produtos }\end{array}$ & $\begin{array}{l}\text { É o tempo que o fornecedor leva para } \\
\text { entregar um pedido. }\end{array}$ & $\begin{array}{l}\text { Data e/ou Hora da realização do } \\
\text { pedido ao fornecedor }- \text { Data e/ou } \\
\text { Hora da entrega dos produtos. }\end{array}$ \\
\hline
\end{tabular}

Fonte: Adaptado de CSCMP, 2019; Garcia-Arca et al., 2018; Ribeiro, 2019; Vieira; Yoshizaki e Ho, 2015.

As organizações devem decidir quais dessas submedidas, apresentadas no quadro 1 (entre tantas outras), são mais apropriadas para serem adotadas. Vieira et al. (2015) concluem que o resultado do desempenho logístico provocam uma aproximação entre os fornecedores e 
clientes, resultando em uma menor incerteza e maior comprometimento.

\subsection{Relacionamento entre integração da cadeia e desempenho de entrega do fornecedor.}

Um melhor gerenciamento da cadeia pressupõe uma maior integração entre os elos (atores) da cadeia e entre as atividades da própria organização, em cada elo individualmente (Croom \& Giannakis, 2000), em prol de aumentar a vantagem competitiva dos próprios atores, por meio da eficiência operacional, resultando na otimização da cadeia como um todo. Portanto, o desempenho de uma empresa será altamente influenciado por seus relacionamentos interfirmas ou alianças estratégicas (Das et al., 2006; Gripa et al., 2019).

O ambiente de negócios globalmente competitivo de hoje coloca pressões nas empresas para melhorar continuamente o atendimento ao cliente, ao mesmo tempo em que reduz os custos e encurta os ciclos de vida dos produtos (Ngniatedema, Chen, \& Guiffrida, 2016), bem como, a importância de a cadeia de suprimentos atender à demanda dos clientes com um desempenho de entrega pontual e confiável (CSCMP, 2019; Ferreira, 2019). O nível de serviço de entrega revela os dados de uma das operações importantes de uma empresa.

O nível de serviço de entrega revela os dados de uma das operações mais importantes de uma empresa: a entrega dos produtos, sendo relevante para entender como está o desempenho da operação da cadeia de suprimentos, possibilitando medir os níveis de eficiência dos processos executados pelos elos da cadeia, mensurando os resultados obtidos e ainda encontrando pontos deficitários para serem corrigidos.

\section{Metodologia de pesquisa}

Trata-se de um estudo descritivo de natureza quantitativa que considera como método de pesquisa o levantamento de informações, cuja coleta de dados foi realizada entre setembro/2018 e dezembro/2018, pelas modalidades on-line e impressa, a um grupo de profissionais atuantes na atividade industrial, comercial, transporte ou prestação de serviço, conforme classificação indicada por Hair, Babin, Money, \& Samouel (2005).

Para tanto, faz uso de levantamento de dados primários por meio da aplicação de questionário estruturado (e-survey) junto aos profissionais que já finalizaram ou estão realizando um curso de aperfeiçoamento em Logística e Cadeia de Suprimentos. Os respondentes exercem funções ligadas a posições de liderança (diretor, gerente, coordenador, supervisor) na empresa; 
Este estudo teve amostra intencional, pois colocou como sujeitos da amostra profissionais que buscaram aperfeiçoamento em curso de especialização na área de Logística e da Cadeia de Suprimentos (Costa Neto, 1977). Foi também uma amostra não probabilística por conveniência devido à inacessibilidade a toda a população.

A amostra foi coletada de parte da população acessível aos pesquisadores, (e-mail de exalunos e existência de turmas em andamento). Participaram da pesquisa profissionais que atuam praticamente em empresas de todas as regiões brasileiras. Foram coletados 243 registros com todos os dados completos, e sem presença de casos extremos multivariados Ao final da fase de coleta, os dados foram tabulados e submetidos ao software Statistical Package for the Social Sciences (SPSS) (IBM, 2016), para a realização do teste Mahalanobis Distances, buscando a identificação de casos extremos multivariados. Para analisar as relações entre os construtos com a Modelagem de Equações Estruturais, com estimação por Mínimos Quadrados Parciais, utilizou-se o software SmartPLS versão 3.2.8 (Ringle, Wende, \& Becker, 2015). No que diz respeito a demografia dos dados coletados, faz-se importante realizar uma correlação entre os resultados das variáveis caracterizadoras.

Os resultados demonstram que 77,40\% (188) dos respondentes possuem entre 30 e 50 anos, $81,10 \%$ atuam a mais de 5 anos na área, $76,90 \%$ possuem um nível de escolaridade de no mínimo Pós-graduação, 88,00\% são gestores - coordenadores, gerentes ou diretores -, podendo-se inferir que são profissionais com maturidade profissional e acadêmica, conhecedores do negócio que administram o que possibilita respostas com viés prático e acadêmico ao instrumento de pesquisa.

Outro ponto importante a ser ressaltado refere-se à área de trabalho e ao setor de atuação dos respondentes, sendo que 44,9\% trabalham na área logística e 94,61\% atuam no setor terciário (comércio, prestação de serviço, transporte). Uma importante correlação entre a área de trabalho e o setor de atuação pode ser observada: conhecimento adequado das questões investigadas neste estudo pelos respondentes, uma vez que quase $50 \%$ do público pesquisado atuam na área logística e quase $95 \%$ no setor terciário, o que pode propiciar uma avaliação, com certa propriedade e domínio pela vivência e expertise profissional, das investigações propostas neste estudo.

As amostras coletadas estão dispersas por 18 Estados brasileiros, com maior expressividade 
na região sudeste $54,75 \%$, de acordo com os respondentes. As organizações onde atuam $67,10 \%$ dos respondentes possuem mais de 500 funcionários, mais de 200 fornecedores $(53,70 \%)$ e mais de 1000 clientes $(55,60 \%)$.

\subsection{Modelo e hipótese da pesquisa}

Tendo em vista a importância dos processos estudados e suas possíveis contribuições para que a cadeia de suprimentos alcance seus objetivos, propõem-se o modelo de pesquisa, apresentado na Figura 1. O modelo foi fundamentado pela junção de dois estudos presentes na literatura.

O primeiro estudo verificou a existência de relação entre a integração da cadeia de suprimentos com os desempenhos organizacionais individuais e globais das cadeias em empresas brasileiras de grande porte (Scandiuzzi, 2011). Este estudo inspirou a proposição do construto integração da cadeia de suprimentos. Contudo, na presente pesquisa procura-se verificar o efeito da relação da integração da cadeia de suprimentos no desempenho de entrega do fornecedor. Para tal, foi verificado os tipos de integração: integração externa com fornecedores, integração interna e integração externa com clientes.

O segundo estudo que fundamentou o modelo foi realizado por Bastos (2012), no qual foi analisada a relação entre as práticas de compartilhamento de conhecimento entre clientes e seus fornecedores de processos industriais e o desempenho de entrega desses fornecedores. Bastos (2012) elencou cinco indicadores, identificados na literatura e em entrevistas realizadas com as empresas pesquisadas, utilizados para avaliar o desempenho de entrega dos fornecedores, sob a perspectiva do cliente.

O modelo referendado na Figura 1 busca compreender as influências da integração da cadeia de suprimentos para alcance de desempenho superior de entrega do fornecedor, sob a perspectiva do cliente. 
Figura 1 - Modelo detalhado da pesquisa

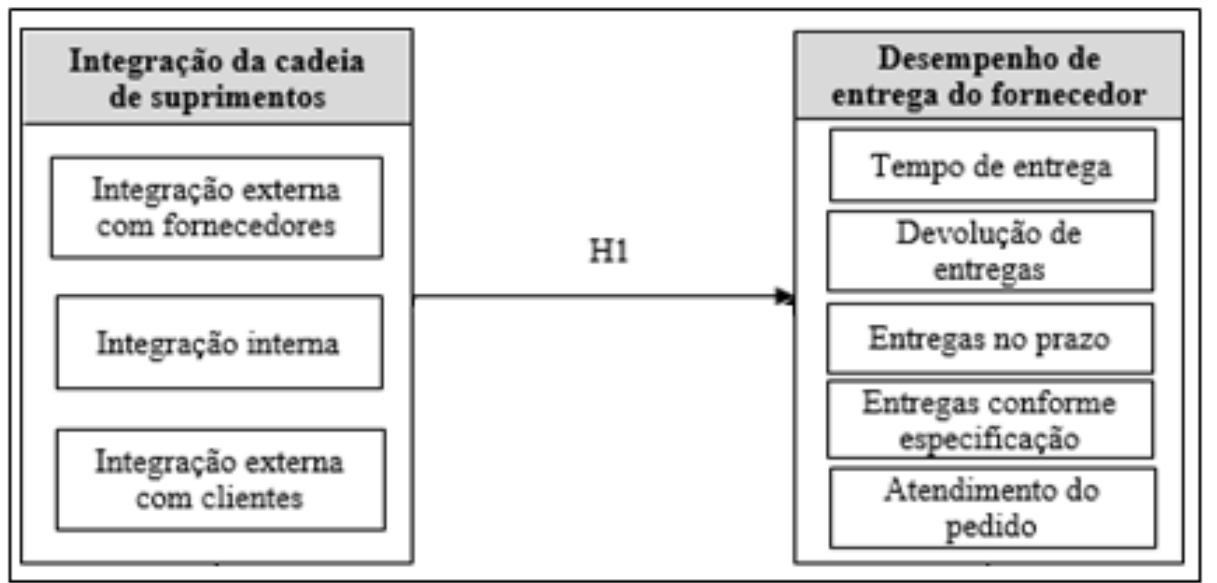

Fonte: Autores, 2020.

O modelo da Figura 1 sugere a existência de relações entre integração da cadeia de suprimentos, composta pela integração externa com fornecedores, integração interna e integração externa com clientes, e desempenho de entrega do fornecedor, composto por tempo de entrega, devolução de entregas, entregas no prazo, entregas conforme especificação e atendimento do pedido. Diversos drivers intangíveis de desempenho são a chave para a obtenção de desempenho superior em termos de entregas.

Para isso, desenvolveu-se a hipótese da pesquisa $\mathrm{H} 1$, a seguir:

- Hipótese 1: a integração da cadeia de suprimentos tem efeito positivo no desempenho de entrega do fornecedor.

Richardson e Urbanke (2008) salientam que hipóteses podem ser definidas como soluções tentativas, previamente selecionadas, do problema de pesquisa, permitindo orientar a análise dos dados, no sentido de aceitar ou rejeitar as soluções tentativas. A integração da cadeia de suprimentos, neste estudo, refere-se à integração da cadeia de suprimentos externa (incluindo integração de fornecedores e integração de clientes) e integração interna.

Relacionamentos colaborativos (Yan \& Azadegan, 2017) com empresas compradoras também podem ser uma fonte de criatividade e novas ideias, bem como uma maneira eficaz de garantir lealdade ao vendedor (Charterina, Landeta, \& Basterretxea, 2017). Nesse sentido, há a necessidade de estabelecer e manter relacionamentos de longo prazo (Gripa \& De Carvalho, 2019) e encontrar mecanismos para difusão eficaz de necessidades e conhecimento entre compradores e fornecedores. A integração da cadeia de fornecimento proporciona aos seus membros oportunidades de comunicação amigáveis, facilitando a construção de consenso, o 
que contribui para o compartilhamento aberto e o uso efetivo do conhecimento tácito. Lee, Ooi, Chong, \& Seow (2014) também descobriram, em seus estudos, que a rede da cadeia de suprimentos era uma importante fonte de conhecimento e recursos para as empresas.

A dimensão de eficiência do desempenho está bem documentada na literatura, mostrando como as rotinas de aprendizado facilitam a eficiência operacional, o corte de custos, ou a implementação efetiva de práticas de fabricação baseadas no tempo (Di Serio \& Vasconcellos, 2017). Esses estudos descobriram que o foco inicial construção de relacionamento integrados (Abbade, 2016; Yan \& Azadegan, 2017) tem sido a melhoria nos processos operacionais interempresariais do dia-a-dia. Devido ao impacto direto que a pontualidade da entrega tem na satisfação do cliente, melhorar o desempenho do processo de entrega é uma preocupação fundamental dos gerentes da cadeia de suprimentos e de logística (Frohlich \& Westbrook, 2001). Não obstante, a entrega de produtos e serviços complexos, quando conhecimento valioso é reconhecido, compartilhado e absorvido pelos parceiros, pode requerer melhorias na capacidade cognitiva da empresa vendedora e nas capacidades de processamento de informações de uma condição de racionalidade limitada para uma de racionalidade especializada (Ribeiro et al., 2019).

\section{Resultados}

O tratamento e validação das variáveis latentes formativas (Integração externa com fornecedores, Integração Interna; Integração externa com clientes) e da variável latente reflexiva (desempenho de entrega) foram segregados em dois modelos, a saber: i) Modelo de Mensuração, que envolve a Integração externa com fornecedores, Integração Interna; Integração externa com clientes; e o desempenho de entrega do fornecedor; ii) Modelo Estrutural, que envolvé as relações existentes as variáveis latentes formativas e a variável latente reflexiva. Nos próximos dois tópicos, foram apresentados a análise dos resultados dos Modelos de Mensuração e Estrutural.

Esta seção apresenta os resultados obtidos sobre os dados, após a aplicação do método de Modelagem de Equações Estruturais, com estimação por Mínimos Quadrados Parciais

\subsection{Modelo de Mensuração}

Pode-se destacar, ao analisar a Tabela 1, que todos os itens apresentaram cargas fatoriais 
maior que 0,70 e não foi necessário retirar nenhum da análise. Vale ressaltar que foi utilizada uma abordagem reflexiva para a construção do modelo. Na Tabela 1, são apresentados os pesos, as cargas fatoriais e as comunalidades do modelo de mensuração.

Tabela 1- Modelo de Mensuração - Abordagem Reflexiva

\begin{tabular}{llccc}
\hline \multicolumn{1}{c}{ Construtos } & \multicolumn{1}{c}{ Itens/Indicador } & Peso $(\boldsymbol{\alpha})$ & C.F. & Com. \\
\hline \multirow{2}{*}{$\begin{array}{llcc}\text { Integração da cadeia } \\
\text { de suprimentos }\end{array}$} & Integração externa com fornecedores & 0,42 & 0,83 & 0,68 \\
& Integração Interna & 0,40 & 0,74 & 0,55 \\
& Integração externa com clientes & 0,43 & 0,82 & 0,66 \\
\hline \multirow{2}{*}{$\begin{array}{l}\text { Desempenho de } \\
\text { entrega de }\end{array}$} & Entrega realizada dentro do prazo negociado & 0,27 & 0,83 & 0,69 \\
fornecedores & Devolução de entregas & 0,26 & 0,80 & 0,64 \\
& Entrega de produtos de acordo com as especificações & 0,29 & 0,78 & 0,62 \\
& Atendimento do pedido realizado & 0,24 & 0,81 & 0,66 \\
& Tempo de entrega de produtos & 0,20 & 0,77 & 0,60 \\
\hline
\end{tabular}

${ }^{1}$ Carga Fatorial; ${ }^{2}$ Comunalidade.

Fonte: Dados da pesquisa, 2019.

A primeira fase consiste em examinar a confiabilidade e a validade dos indicadores e construtos, com base em determinados critérios associados à especificação do modelo de mensuração (Ringle et al., 2015). A confiabilidade pode ser verificada pela análise do Alfa de Cronbach sobre valores que variam de 0 a 1 (Cronbach, 1951). Essa abordagem tem por objetivo analisar a ausência de erro aleatório nas unidades de mensuração da escala. Quanto mais próximo de 1 , maior a evidência da confiabilidade do conjunto de itens. É possível notar que os indicadores possuem consistência interna, uma vez que as medidas que refletem a confiabilidade composta são superiores a 0,70 (Malhotra, 2012). Ou seja, os construtos são confiáveis, conforme apresentado na Tabela 2.

Tabela 2 - Validação do modelo de mensuração - Abordagem Reflexiva

\begin{tabular}{lcccccc}
\hline \multicolumn{1}{c}{ Construtos } & Itens & A.C. $^{\mathbf{1}}$ & C.C. $^{\mathbf{2}}$ & Dim. $^{\mathbf{3}}$ & $\mathbf{A V E}^{\mathbf{4}}$ & VCM $^{\mathbf{5}}$ \\
\hline Integração da cadeia de suprimentos & 3 & 0,708 & 0,837 & 1 & 0,633 & 0,486 \\
$\begin{array}{l}\text { Desempenho de entrega de } \\
\text { fornecedores }\end{array}$ & 5 & 0,860 & 0,899 & 1 & 0,640 & 0,129 \\
\hline
\end{tabular}

${ }^{1}$ Alfa de Cronbach,${ }^{2}$ Confiabilidade Composta, ${ }^{3}$ Dimensionalidade, ${ }^{4}$ Variância Extraída; ${ }^{5}$ Variância Compartilhada Máxima.

Fonte: Dados da pesquisa, 2019.

A análise da validade convergente, a validade discriminante, a dimensionalidade e a confiabilidade dos construtos do modelo de mensuração final, demonstradas na Tabela 2, possibilitou destacar que: i ) em todos os construtos, os índices de confiabilidade A.C. ou C.C. apresentaram valores acima de 0,70, evidenciando, assim, a confiabilidade dos mesmos; pelo 
critério de Kaiser, todos os construtos foram unidimensionais; ii) todas as AVEs foram superiores a 0,40 , indicando que houve validação convergente em todos os construtos; iii) pelo critério proposto por Fornell e Larcker (1981) houve validação discriminante para todos os construtos, uma vez que as variâncias compartilhadas máximas foram menores que as respectivas AVEs.

Estimativas válidas e confiáveis do modelo de mensuração permitem avaliar o modelo estrutural (Henseler, Ringle, \& Sinkovics, 2009). Considerados satisfatórios os resultados, no que se refere à confiabilidade e à validade (convergente e discriminante) dos indicadores e construtos, inicia-se a análise das relações entre os construtos.

\subsection{Modelo Estrutural}

A segunda fase na PLS-SEM implica em estabelecer a capacidade de predição e analisar os relacionamentos entre os construtos. Os critérios de avaliação compreendem o nível de significância dos coeficientes do caminho estrutural e o coeficiente de determinação R2. O resultado do modelo estrutural é apresentado na Tabela 3.

Tabela 3 - Modelo Estrutural

\begin{tabular}{lccccc}
\hline \multicolumn{1}{c}{ Endógena } & Exógena & $\boldsymbol{\beta}$ & I.C. - 95\% & Valor-p & $\mathbf{R}^{\mathbf{2}}$ \\
\hline $\begin{array}{l}\text { Desempenho de entrega } \\
\text { de fornecedores }\end{array}$ & $\begin{array}{l}\text { Integração da cadeia } \\
\text { de suprimentos }\end{array}$ & 0,132 & {$[-0,04 ; 0,29]$} & 0,160 & $15 \%$ \\
\hline
\end{tabular}

\section{${ }^{1}$ Erro Padrão;}

Fonte: Dados da pesquisa, 2019.

Na análise do modelo estrutural (Tabela 3), o foco está na explicação dos construtos dependentes, e o interesse fundamental é que o coeficiente de determinação $\mathrm{R}^{2}$ seja elevado (Hair Jr, Hult, Ringle, \& Sarstedt, 2016). Esse coeficiente é uma medida de precisão preditiva do modelo, e seus valores variam de 0 a 1 . Níveis elevados indicam maior precisão.

A soma dos efeitos diretos e indiretos é referida como o efeito total, e a análise relativa da importância das relações é fundamental para a interpretação dos resultados e para as conclusões (Hair Jr et al., 2016). O efeito total dos coeficientes no caminho estrutural é apresentado na Tabela 4. 
Tabela 4 - Análise do Efeito Direto, Indireto e total dos construtos do modelo

\begin{tabular}{llccc}
\hline \multicolumn{1}{c}{ Endógena } & \multicolumn{1}{c}{ Exógena } & Efeito Direto & Efeito Indireto & Efeito Total \\
\hline $\begin{array}{l}\text { Desempenho de entrega } \\
\text { de fornecedores }\end{array}$ & $\begin{array}{l}\text { Integração da cadeia } \\
\text { de suprimentos }\end{array}$ & 0,132 & 0,205 & 0,337 \\
\hline \hline
\end{tabular}

Fonte: Dados da pesquisa, 2019.

A ilustração do modelo estrutural é demonstrada na Figura 2.

Figura 2 - Ilustração do Modelo Estrutural

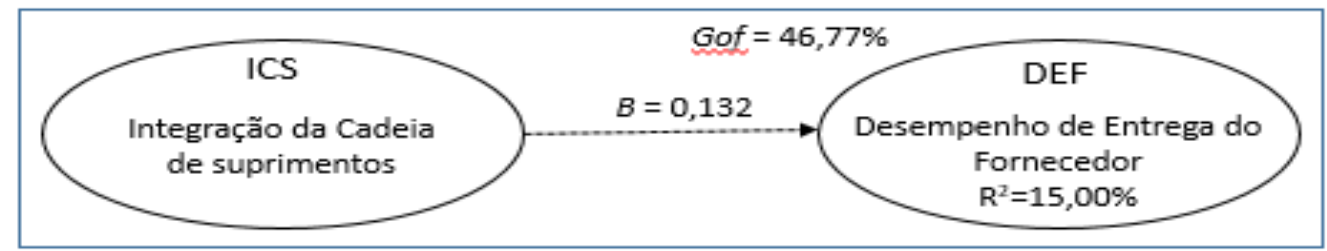

Fonte: Dados da pesquisa, 2019.

A análise dos resultados do modelo estrutural, apresentados nas Tabelas 3 e 4 e na Figura 2, possibilitou concluir que em relação ao Desempenho de entrega de fornecedores não houve influência significativa (valor- $p=0,160)$ e positiva $(\beta=0,132)$ da Integração da cadeia de suprimentos sobre o Desempenho de entrega de fornecedores. Logo, a Integração da cadeia de suprimentos não influencia o Desempenho de entrega de fornecedores.

Com isso, conclui-se a análise dos resultados com o método PLS-SEM, e as hipóteses expressas pelo modelo hipotético podem ser consideradas: suportadas ou rejeitadas. No próximo tópico, foi apresentado a avaliação das análises.

\subsection{Avaliação da Hipótese}

A hipótese foi formulada tendo por base os construtos propostos neste estudo, os achados de alguns estudos empíricos, a revisão de literatura apresentada e, a partir de uma estimativa e de uma resposta presumida e provisória, previamente, que, de acordo com os critérios estabelecidos, serão rejeitadas ou suportadas. De acordo com os dados exibidos nas Tabelas 3 e 4 e na Figura 2, foi possível realizar a avaliação das hipóteses da pesquisa.

A Figura 3 apresenta a síntese dos resultados do teste da Hipótese do estudo. 
Figura 3 - Síntese dos Resultados

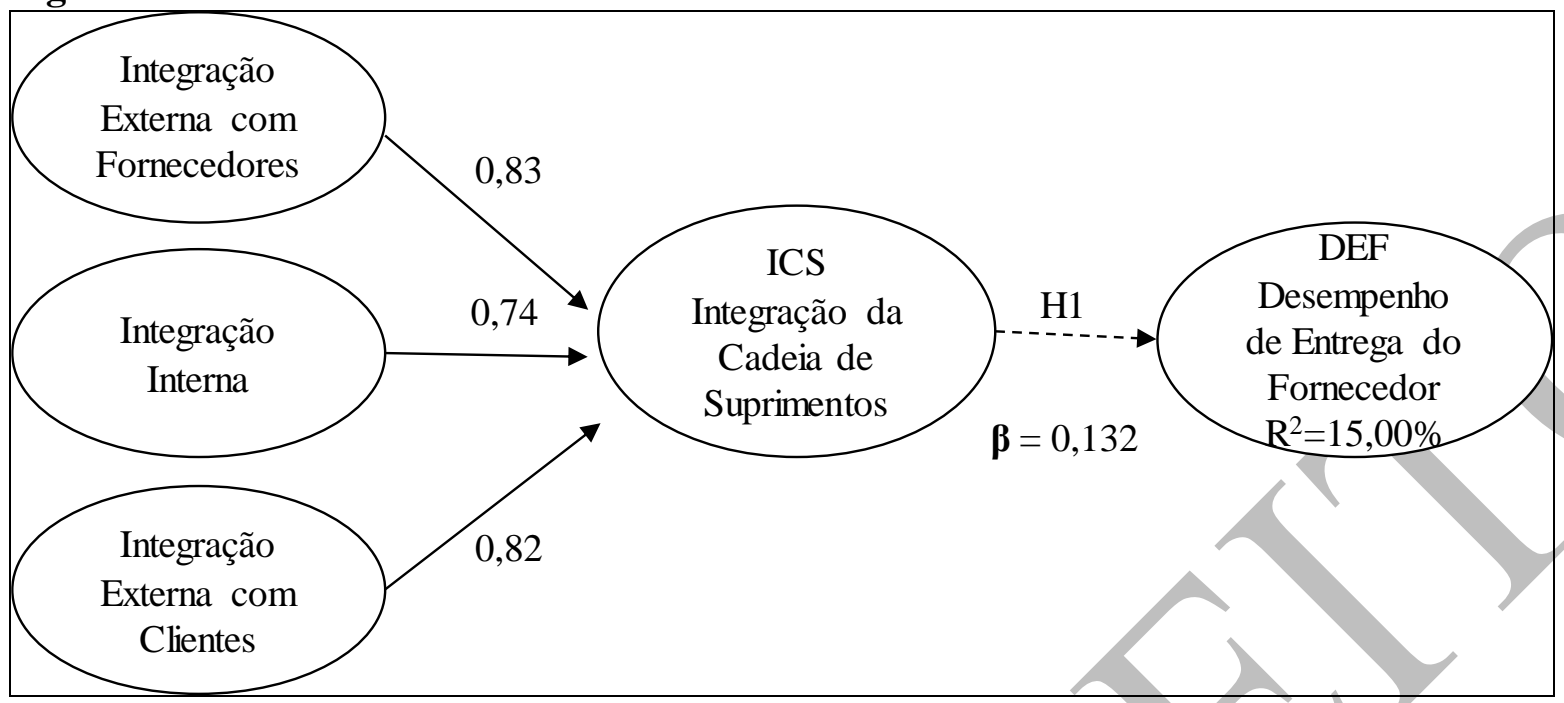

Fonte: Dados da pesquisa, 2019.

A hipótese $\mathbf{H 1}$ sugere que a Integração da Cadeia de Suprimentos (ICS) tem efeito positivo no Desempenho de Entrega do Fornecedor (DEF). (ICS -> DEF). Seu teste revela que não houve influência significativa (valor- $p=0,160)$ e positiva $(\beta=0,132)$ da Integração da cadeia de suprimentos sobre o Desempenho de entrega de fornecedores (Tabela 3 e Figura 3), cumprindo o objetivo geral. Logo, a Integração da cadeia de suprimentos não influencia o Desempenho de entrega de fornecedoresEsse resultado implicou em não confirmar a hipótese H1.

O fato de não existir confirmação da hipótese H1, conforme resultado do teste da hipótese sugere discussão das relações entre os construtos de primeira ordem e das relações dos construtos de segunda ordem.

Salienta-șe que há uma comprovação estatística da importância da composição dos constructos de primeirá ordem: Integração externa com fornecedores (IF), integração interna (II) e integração externa com clientes (IC) (relacionados a ICS) e tempo de entrega, devolução de entregas, entregas no prazo, entregas conforme especificação e atendimento do pedido (relacionados a DEF), quando se mantêm análises de Integração da Cadeia de Suprimentos e Desempenho de Entrega do Fornecedor.

Os escores da carga fatorial quanto à integração externa com fornecedores e a integração externa com clientes encontram-se próximos; porém, há maior influência da integração externa com fornecedores $(0,83)$, seguida pela integração com clientes $(0,82)$, e, por fim, pela integração interna $(0,74)$. Isso demonstra que os respondentes julgaram que a integração 
externa, seja com clientes, seja com fornecedores, favorece a ICS de forma mais significativa do que a integração interna, de acordo com o resultado encontrado na Tabela 1.

Tais resultados também confirmam a literatura estudada. Para Scandiuzzi (2011) e Zailani e Rajagopal (2005) existem categorias que afetam o desempenho da integração da cadeia de suprimentos, dentre elas estão: integração interna - aborda a integração geral de todas as funções internas e departamentos da empresa; integração externa com fornecedores - pela integração com os fornecedores, é verificado o quão próximo as empresas estão trabalhando.

Devem ser abordados o nível de envolvimento e influência dos fornecedores nas decisões da empresa, e também o nível de parceria estratégica adotada (grau de parceria, formal ou informal, que a empresa faz com seus fornecedores); e na integração externa com consumidores - de forma parecida com o item anterior, pela integração com os fornecedores, é verificado o quão próximo as empresas estão trabalhando. Devem ser abordados o nível de envolvimento e influência dos clientes nas decisões da empresa e também as respostas (feedback) dos clientes aos produtos lançados pela empresa e as ações das empresas com relação às necessidades e às reclamações dos clientes.

Os construtos de segunda ordem no modelo são constituídos pelos construtos Integração da Cadeia de Suprimentos (ICS) e Desempenho de Entrega do Fornecedor (DEF). Os construtos de segunda ordem fornecem uma melhor compreensão teórica das relações propostas no modelo da Figura 2. A relação direta Integração da Cadeia de Suprimentos e Desempenho de entrega do fornecedor (ICS -> DEF) apresentou resultado não significante, ou seja, o resultado mostra que não há influência direta de ICS em DEF, rejeitando H1.

Este resultado, por um lado, é incoerente com a literatura pesquisada. Como por exemplo, Frohlich e Westbrook (2002), realizaram uma pesquisa com quatrocentas e cinco empresas do Reino Unido, e foi evidenciado que as empresas integradas com clientes (integração da previsão da demanda, dados do perfil do cliente, recebimento de pedidos on-line e gerenciamento do relacionamento com o cliente) e fornecedores (planejamento de estoque, previsão da demanda e agendamento de pedidos integrados) possuem um maior nível de desempenho (velocidade de entrega, redução de custos de transação, aumento do giro de estoque e maior rentabilidade.

Por outro lado, o resultado encontrado nesta pesquisa coincidiu com os estudos dos autores 
Badell et al. (2007), Das et al. (2006) e Swink et al. (2007) que mostraram que os benefícios da integração da cadeia de suprimentos não puderam ser confirmados, mesmo com diversos benefícios atribuídos às ações de integração com outros elos da cadeia, por estudos acadêmicos e relatórios de mercado.

A realidade encontrada neste estudo da inexistência da relação direta ICS -> DEF pode ser justificada pela dificuldade das organizações, de grande parte dos respondentes, não estarem motivadas ou não estarem aptas a perceber ou realizar a relação de interdependência entre as empresas (relação cliente e fornecedor). Já que a implementação da integração da cadeia requer que as empresas implementem ações, objetivando tanto a integração interna quanto a integração externa (Scandiuzzi, 2011; De Abreu Siqueira \& Chicarelli Alcântara, 2020) a fim de aumentarem o desempenho de entrega. Para realização deste relacionamento integrativo alguns aspectos devem ser considerados como: acesso aos sistemas de planejamento entre as empresas; compartilhamento dos planos de produção; conhecimento dos níveis e do mix de estoque; customização de embalagens; frequências de entregas; uso comum de equipamentos logísticos e uso comum de serviços prestados por operadores logísticos, atendimento à demanda dos clientes com um desempenho de entrega pontual (Mancio \& Sellitto, 2017).

Scandiuzzi (2011) mencionou, em seus estudos, uma questão importante sobre os desempenhos empresariais individuais e desempenhos globais das cadeias, questionando se a prática comum de cada empresa pertencente a uma cadeia na busca da sua própria otimização de resultados não leva a uma melhora nos resultados da cadeia como um todo. Ou seja, a otimização das partes não traz a otimização do todo.

Os estudos citados demonstram que iniciativas são empreendidas por algumas empresas, porém poucos são os rêsultados efetivos, justificando, em parte, os achados desta pesquisa quanto a rejeição de $\mathrm{H} 1$, em que maior parte dos respondentes não percebem a influência que a Integração da cadeia de suprimentos tem sobre o construto desempenho de entrega do fornecedor. Um aspecto importante a ser levado em consideração diz respeito à área em que trabalham os respondentes, como possível explicação do resultado obtido de rejeição de H1. Apesar da área de logística ter sido responsável por 44,9\%, o percentual de respondentes que trabalham em outra área diferente das áreas de logística, inovação, produção e comercial, corresponde a 35,8\%. Esse percentual de respondentes pode desconhecer os resultados das relações propostas no modelo deste estudo, por não terem ligação direta com os mesmos. Em 
outras palavras, os respondentes não percebem a importância da integração da cadeia de suprimentos sobre o desempenho de entrega do fornecedor por não terem suas atividades influenciadas pelas relações dos construtos citados.

Outro elemento plausível de ser influenciador da rejeição da hipótese H1 pode ser advindo da maior representatividade dos setores de atuação das empresas, nas quais trabalham os respondentes; corresponderem ao transporte $(75,7 \%)$ e prestação de serviço $(18,5 \%)$, totalizando $64,2 \%$ da amostra. A pequena participação de respondentes do setor de indústria $(1,66 \%)$, pode ter influenciado no resultado, uma vez que a área de indústria está totalmente inserida no contexto da cadeia de suprimentos como área central do processo que envolve os construtos pesquisados. A indústria é afetada diretamente pelos impactos da integração da cadeia de suprimentos por sofrer influência tanto da eficácia de fornecedores quanto de informações dos clientes para um desempenho de entrega satisfatório.

Assim, esta pesquisa propôs um modelo de análise válido e confiável, apesar da rejeição da hipótese da pesquisa. Também possibilitou identificar todos os desafios aventados nas relações entre os construtos, e tantos outros que podem surgir para a ocorrência da integração da cadeia de suprimentos, reside aí a dificuldade das organizações da maioria dos respondentes de adoção destas práticas, como possíveis justificativas da rejeição de H1. Vale ressaltar que as organizações podem até perceber a importância e os benefícios advindos da adoção da integração da cadeia para o aumento do desempenho de entrega; contudo, ainda não conseguiram implementar esta evolução. Ou até mesmo podem tê-las, mesmo que de modo incipiente, porém, os funcionários ainda não conseguiram perceber.

Além do mais, a entrega, por sua própria natureza, ocorre em um ambiente dinâmico e em constante mudança, difícultando o estudo e a melhoria subsequente de um sistema de distribuição. A antecipação de como e quando estas mudanças ocorrerão trata-se de um dos principais elementos a serem dominados pelos intervenientes da cadeia dentro de uma estrutura de distribuição, e tal dinâmica afetará o sistema como um todo. A integração da cadeia de suprimentos pode atuar nessa seara, de modo a minimizar as situações inesperadas e intempestivas requeridas no desenvolvimento de entrega superior ao fornecedor.

Realizando-se uma análise do perfil dos respondentes, pode-se inferir que são profissionais com certa maturidade profissional e acadêmica, conhecedores do negócio que administram, o que possibilita respostas com viés prático e acadêmico ao instrumento de pesquisa. As 
amostras coletadas se encontram dispersas por 18 Estados brasileiros, podendo-se inferir uma capilaridade interessante do estudo.

\section{CONCLUSÃO}

O nível de desempenho de uma organização pode estar diretamente ligado ao modo como ela desempenha a tarefa de gerenciamento de seus processos internos, à forma como domina competências e habilidades internas de maneira eficiente, bem como à forma como ela estabelece suas relações com seus fornecedores, visando a atender aos requisitos dos clientes, resultando na agregação de valor ao produto ou serviço oferecido. Contudo, apesar de ser possível um entendimento intuitivo acerca das relações da integração entre empresas da cadeia de suprimentos para obtenção de um desempenho de entrega superior, observam-se algumas lacunas e oportunidades de pesquisa. Faz-se importante compreender como o desenvolvimento de um ambiente propício, entre os intervenientes da cadeia de suprimentos, pode auxiliar no alcance de um desempenho de entrega ao cliente satisfatório.

Nessa perspectiva, a problemática deste estudo teve a seguinte questão de pesquisa: Quais são as influências da relação entre integração da cadeia de suprimentos para alcance de um desempenho superior de entrega do fornecedor? Para auxiliar na investigação, a metodologia de pesquisa empreendida foi a abordagem quantitativa e descritiva quanto aos fins. Quanto aos procedimentos, foi realizado por intermédio de um levantamento (survey) transversal auto administrado on-line e impresso, aplicado a um grupo de profissionais atuantes na atividade industrial, comercial, transporte ou prestação de serviço. Como ferramenta de tratamento e análise dos dados dos questionários foi utilizado o software SPSS (Statistic Package for Social Study) e a Modelagem de Equações Estruturais, com estimação por Mínimos Quadrados Parciais (PLS-SEM).

O objetivo geral deste estudo foi alcançado, bem como os objetivos específicos propostos conforme a seguir: 1) foi possível identificar na literatura os tipos de integração da cadeia de suprimentos (Tópico 2.1) e os indicadores mais utilizados para avaliar o desempenho de entrega dos fornecedores, sob a perspectiva do cliente (Tópico 2.2);2) o tópico 3 descreve as relações entre os construtos de primeira e segunda ordem propostas no estudo, e ilustra como ocorreram a criação de cada construto e o desenvolvimento da hipótese. Tomando por base as orientações causais postuladas entre os construtos do modelo, a hipótese H1 foi elaborada, sugerindo que a integração da cadeia de suprimentos tem efeito positivo no desempenho de 


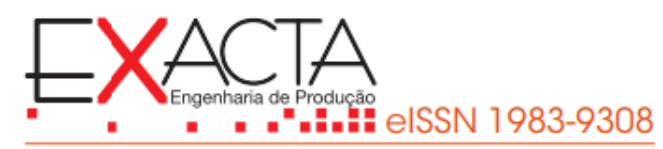

entrega do fornecedor; 3) o modelo proposto na Figura 1 sugere a existência de relações entre dois construtos: integração da cadeia de suprimentos e desempenho de entrega do fornecedor. Este modelo foi fundamentado pela junção de dois estudos realizados presentes na literatura, Scandiuzzi (2011) e Bastos (2012), que instigaram a proposição dos construtos de primeira e segunda ordem, conforme informado no tópico 3. Para a validação do modelo da pesquisa utilizou-se de questionário para obtenção dos dados, tabulação das respostas e análise confirmatória da pesquisa por meio de procedimentos estatísticos, através da Modelagem de Equações Estruturais com estimação por Mínimos Quadrados Parciais (PLS-SEM).

Como resultado da pesquisa, obteve-se que o modelo proposto na Figura 1 é válido e confiável, e, de acordo com os dados exibidos na Figura 2 e Tabelas 3 e 4, foi realizada a avaliação da hipótese da pesquisa. A hipótese da pesquisa não foi confirmada, apesar da dependência entre os construtos de primeira ordem se relacionarem positivamente e estatisticamente significantes, conforme apresentado na Figura 3. Este resultado demonstra que os tipos de integração da cadeia de suprimentos e os indicadores para avaliar o desempenho de entrega dos fornecedores apresentaram influência direta nos construtos respectivos, isoladamente, confirmando a literatura estudada (Bastos, 2012; Scandiuzzi, 2011).

Diante de todo o contexto de resultados da pesquisa apresentado, a questão norteadora da pesquisa foi respondida considerando a percepção dos respondentes. No que diz respeito ao requisito importância ou relevância, esta pesquisa ratificou que os indicadores propostos para medir o desempenho de entrega do fornecedor possuem resultados significantes. Não obstante ainda, atestou que os tipos de integração recomendados influenciam de forma direta a integração da cadeia de suprimentos.

Ao término de realizar esse estudo constatou-se que várias foram as contribuições relevantes do ponto de vista teórico e empírico. No campo teórico pode-se apontar: i) a integração externa, seja com clientes, seja com fornecedores, favorece a integração da cadeia de suprimentos de forma mais significativa do que a integração interna; ii) o estudo corroborou com as pesquisas existentes na literatura permitindo a reflexão a acerca dos construtos integração da cadeia de suprimentos e desempenho de entrega do fornecedor.

As contribuições no campo empírico foram: i) a pesquisa proporcionou um levantamento das principais características de um grupo de 243 respondentes pertencentes a 18 Estados 


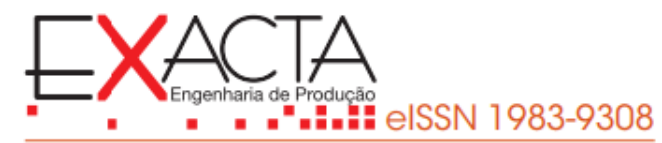

brasileiros; ii) o modelo proposto neste estudo permite que as organizações atuem nos fatores identificados de modo a desenvolver suas capacidades estratégicas, de tal forma que sejam desenvolvidas competências únicas para obtenção e fortalecimento de vantagens sustentáveis da rede de empresas formadas; iii) as influências das relações entre integração da cadeia de suprimentos e desempenho de entrega do fornecedor não foram verificadas na percepção de respondentes que atuam no transporte $(75,7 \%)$, e na prestação de serviço $(18,5 \%)$, o que oportuniza a realização de estudos aprofundados e específicos no setor terciário (transportes e prestação de serviços) para diagnosticar as possíveis causas das relações entre os construtos pesquisados no modelo de análise proposto não terem sido suportadas, já que diversos estudós teóricos e empíricos atestam o contrário conforme referencial teórico apresentado; iv) Também propicia a realização de pesquisa exclusivamente na área de atuação logística e no setor industrial para conhecer como se comportaria as hipóteses $\mathrm{H} 1$.

Este trabalho analisou empresas focais isoladamente (não foram mapeadas as cadeias as quais elas pertencem) através da percepção dos empregados que nela atuam e, portanto, os resultados relativos à cadeia como um todo foram obtidos com base nas opiniões dos executivos dessas empresas focais, o que pode ser considerado uma limitação deste estudo.

Como sugestão para trabalhos futuros seria importante a realização de estudos qualitativos para conhecer melhor as especificidades de cada empresa e de sua respectiva cadeia, o que pode vir a contribuir para o debate e estudo dessa natureza.

\section{REFERÊNCIAS}

Abbade, E. B. (2016). Interorganizational Alignment of Strategic Orientations in Supply Chains. Revista de Negócios, v. 20, n. 2, p. 15-30, 2016. doi: https://bit.ly/3o4Kane

Badell, M., Fernández, E., Guillén, G., \& Puigjaner, L. (2007). Empowering financial tradeoff with joint financial and supply chain planning models. Mathematical and computer modelling, 46(1-2), 12-23. doi: https://bit.ly/2JnJx9o

Bastos, A. L. A. (2012). O efeito do compartilhamento de conhecimentos sobre o desempenho de entrega dos fornecedores em cadeias de suprimentos. Tese (Doutorado em Administração). Universidade Federal de Santa Catarina. Florianópolis.

Bowersox, D. J., Closs, D. J., Cooper, M. B. , \& Bowersox, J. C. (2016). Gestão Logística da Cadeia de Suprimentos, 4 eds., Porto Alegre: Bookman.

Castells, M.(2002). A sociedade em Rede - a era da informação: economia, sociedade e cultura - Volume 1. São Paulo: Paz e Terra. 
García-Arca, J., Prado-Prado, J. C., \& Fernández-González, A. J. (2018). Integrating KPIs for improving efficiency in road transport management. International Journal of Physical Distribution \& Logistics, v.48, n.9, p. 931-951. doi: https://bit.ly/31maI1k

Gripa, S., \& De Carvalho, L. C. (2019). Integração Externa e Capacidade de Inovação Organizacional Moderada pelo Nível de Confiança. Revista Eletrônica de Ciência Administrativa, v.18, n.1, p. 85-104. doi: https://bit.ly/3o7VwXK

Hair, J., Babin, B., Money, A., \& Samouel, P. (2005). Fundamentos de métodos de pesquisa em administração. Bookman Companhia Ed.

Hair Jr, J.F., Hult, G.T.M., Ringle, C., \& Sarstedt, M. (2016). Um primer na modelagem de equações estruturais de mínimos quadrados parciais (PLS-SEM). Publicações Sage.

Henseler, J., Ringle, C. M., \& Sinkovics, R. R. (2009). The use of partial least squares path modeling in international marketing. Advances in International Marketing, v. 20, n. 1, p. 277-319. doi: https://bit.ly/3lhW4YX

IBM. (2016). IBM SPSS statistics 24 core system user's guide. 24. ed. Armonk: IBM Corporation.

Lee, H. L., \& Whang, S. (2001). E-business and supply chain integration. Stanford Global Supply Chain Management Forum, Nov. doi: https://bit.ly/3fKEL1M

Lee, V. H., Ooi, K. B., Chong, A. Y. L., \& Seow, C. (2014). Creating technological innovation via green supply chain management: An empirical analysis. Expert Systems with Applications, 41(16), 6983-6994. doi: https://bit.ly/2KQphxQ

Malhotra, N. K. (2012). Pesquisa de marketing: Uma orientação aplicada. 6. ed., Porto Alegre: Bookman.

Mancio, V. G., \& Sellitto, M. A. (2017). Sistemas flexíveis de manufatura: definições e quadro de trabalho para futura pesquisa. Revista GEINTEC-Gestão, Inovação e Tecnologias, v. 7, n. 2, p. 3760-3773. doi: https://bit.ly/36k7p6U

Ngniatedema, T., Chen, L., \& Guiffrida, A. L. (2016). A modelling framework for improving supply chain delivery performance. International Journal of Business Performance and Supply Chain Modelling, v. 8, n. 2, p. 79-96. doi: https://bit.ly/3odl9q9

Ribeiro, J. S. de A. N. (2019). Modelo de análise do compartilhamento de conhecimento e inovação no desempenho de entrega: um estudo sobre Integração da Cadeia de Suprimentos. Tese. Tese de doutorado, Programa de Pós-Graduação em Sistemas de Informação e Gestão do conhecimento da Universidade FUMEC, Belo Horizonte, MG, Brasil.

Ribeiro, J. S. D. A. N., Ziviani, F., Tadeu, H. F. B., \& De Ramos Neves, J. T. (2019). Gestão do conhecimento e sistemas de informação na cadeia de suprimentos global. RBBD. Revista Brasileira de Biblioteconomia e Documentação, v. 15, n .2, p. 251-289. doi: https://bit.ly/37jatPN 
Ribeiro, J. S. D. A. N., Calijorne, M. A. S., Jurza, P. H., \& Ziviani, F. (2018). The articulation between innovation and competences anchored by knowledge management aiming sustainable competitive advantage. Brazilian Journal of Information Science: Research Trends, v. 12, n. 2. doi: https://bit.ly/33s2Kxw

Richardson, T., \& Urbanke, R. (2008). Teoria da codificação moderna. Cambridge University Press.

Ringle, C. M., Wende, S., \& Becker, J. M. (2015). SmartPLS 3. Bönningstedt: SmartPLS $\mathrm{GmbH}$.

Scandiuzzi, F. (2011). Integração da cadeia de suprimentos no Brasil: um estudo em empresas de grande porte. Tese (Doctoral dissertation), Universidade de São Paulo. São Paulo.

Sezen, B. L. (2008). Relative effects of design, integration and information sharing on supply chain performance. Supply Chain Management, v. 13, n. 3, p. 233-240. doi:

https://bit.ly/39uQmAP

Slack, N., Alistair B. J., \& Johnston, R. (2018). Administração da Produção. 8. ed. São Paulo: Atlas.

Swink, M., Narasimhan, R., \& Wang, C. (2007). Managing beyond the factory walls: effects of four types of strategic integration on manufacturing plant performance. Journal of operations management, 25(1), 148-164. doi: https://bit.ly/3mmnXAq

Viana, F. L. E., Neto, J. D. P. B., Añez, M. E. M., \& Fernandes, J. A. L. (2016). Fontes de obtenção de vantagem competitiva em empresas industriais: uma análise nas indústrias têxtil e de calçados do Ceará. Revista Econômica do Nordeste, Fortaleza, v. 43, n. 3, p. 533-560. doi: https://bit.ly/3mfD52F

Vieira, J. G. V., Yoshizaki, H. T. Y., \& Ho, L. L. (2015). The Effects of Collaboration on Logistical Performance and Transaction Costs. Int. Journal of Business Science and Applied Management, v.10, n.1. doi: https://bit.ly/2Jn8Ubv

Villena, V. H., Choi, T, \& Revilla, E. (2012). Revisiting interorganizational trust: is more always better or could more be worse? Working paper, v.45, n. 2, p. 752-785. doi:

https://bit.ly/3lotSno

Zailani, S., \& Rajagopal, P. (2005). Supply chain integration and performance: US versus East Asian companies. Supply Chain Management, v. 10, n.5, p. 379-393. doi: https://bit.ly/3fMyD9b

Yan, T., \& Azadegan, A. (2017). Comparing inter-organizational new product development strategies: Buy or ally; Supply-chain or non-supply-chain partners? International Journal of Production Economics, v. 183, p. 21-38. doi: https://bit.ly/2HOCyFU 